MAGNETIC-MEMS

\section{A human touch}

Electronic skins are flexible membranes which aim to mimic the functionalities of human skin for use in robotics or virtual reality applications. Typically, these skins are sensitive only to either tactile or touchless inputs, because combining the technology to respond to both has been a challenge. Writing in Nature Communications, Denys Makarov and colleagues present an electronic skin which can identify both tactile and touchless inputs simultaneously within one wearable platform.

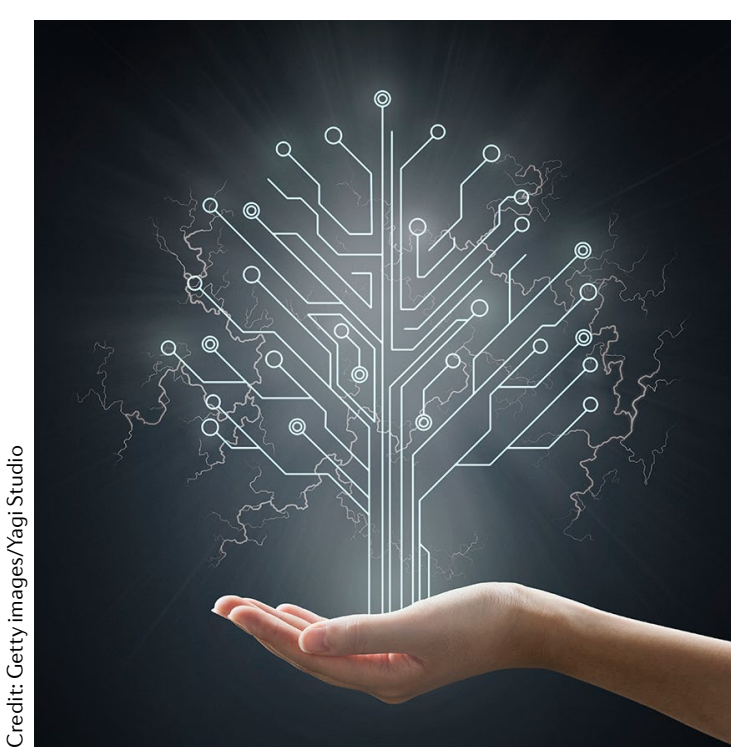

Current sensor technology for wearables uses a variety of parameters to detect an input: humidity, temperature, capacitance or light. However, interference from ambient noise often masks the desired input signal. Makarov and the team use magnetic fields as their detection parameter. Makarov explains that "most surrounding objects are non-magnetic. In this respect, using magnetic field sensors for interaction allows us to assure selectivity in a very easy way: we just need to decorate any object of interest with a magnetic label".

This sensor consists of a $20-\mu \mathrm{m}$ thin flexible polymeric foil and a permanent magnet with pyramidshaped extrusions. The sensor changes its electrical resistance when either of these components is exposed to a magnetic field. The thin foil detects the in-plane external field of a magnetically functionalized object, owing to the giant magnetoresistance effect, and thus is sensitive to touchless interactions. Tactile interactions are detected when a magnetic field is generated from mechanical deformations of the pyramid structures on the permanent magnet.

The biggest challenge is to discriminate between tactile and touchless modes in real time.
"This distinction became possible only when we accounted for particular physiological qualities of humans in the interaction process," explains Makarov. Our fingers have limited sensitivity, and can only perceive pressures of about $300 \mathrm{~Pa}$. In addition, we cannot control our fingers precisely. It is difficult to hold a finger steady $1 \mathrm{~mm}$ away from a surface without touching it. "Combining these human-specific aspects allowed us to put forth an efficient algorithm of how to carry out the interpretation of the signal and assign it to the touchless or tactile interactions unambiguously" says Makarov.

The next steps are to develop interactions with multiple magnetic objects, with different field strengths, symmetries or field gradients, and be able to uniquely identify them. Furthermore, the researchers envisage designing a magnetic sensor to detect generic stray fields independent of their orientation in space. Makarov hopes to "combine Hall effect and magnetoresistive sensors in a single sensor unit which will pave the way towards the realization of omnidirectional magnetosensitive electronic skins. This is one of the major milestones in the field of shapeable magnetoelectronics."

Ankita Anirban

ORIGINAL ARTICLE Ge et al. A bimodal soft electronic skin for tactile and touchless interaction in real time. Nature Communications 10, 4405 (2019) 